\title{
Using a Spectrofluorometer for Resonance Raman Spectra of Organic Molecules
}

\author{
Vadivel Masilamani, ${ }^{1,2}$ Hamid M. Ghaithan, ${ }^{1}$ Mamduh J. Aljaafreh, ${ }^{1}$ Abdullah Ahmed, ${ }^{1}$ \\ Reem al Thagafi, ${ }^{1}$ Saradh Prasad, ${ }^{1,2}$ and Mohamad S. Alsalhi ${ }^{1,2}$ \\ ${ }^{1}$ Department of Physics and Astronomy, College of Science, King Saud University, P.O. Box 2455, Riyadh 11451, Saudi Arabia \\ ${ }^{2}$ Research Chair on Laser Diagnosis of Cancers, Department of Physics and Astronomy, College of Science, King Saud University, \\ Riyadh 11451, Saudi Arabia
}

Correspondence should be addressed to Vadivel Masilamani; masila123@gmail.com

Received 8 October 2016; Accepted 5 January 2017; Published 21 March 2017

Academic Editor: Jau-Wern Chiou

Copyright (c) 2017 Vadivel Masilamani et al. This is an open access article distributed under the Creative Commons Attribution License, which permits unrestricted use, distribution, and reproduction in any medium, provided the original work is properly cited.

\begin{abstract}
Scattering (Rayleigh and Raman) and fluorescence are two common light signals that frequently occur together, confusing the researchers and graduate students experimenting in molecular spectroscopy laboratories. This report is a brief study presenting a clear discrimination between the two signals mentioned, employing a common spectrofluorometer such as the PerkinElmer LS 55. Even better, the resonance Raman signal of a molecule (e.g., acetone) can be obtained elegantly using the same instrument.
\end{abstract}

\section{Introduction}

When photons fall on organic molecules (benzene, acetone, coumarin, etc.), some get scattered, while others are absorbed. Among the scattered signals, some have the same wavelength of the incident photon (termed Rayleigh scattering signals), some have wavelength longer than the incident light (called Raman Stokes signal), and only very few photons have a shorter wavelength (called Raman anti-Stokes signal) [1].

Some molecules which absorb the photons produce fluorescence; this is a reemission process, a phenomenon from one quantum state to another one. A molecule (i.e., acetone) is at the ground state electronic level $\mathrm{S}_{0}$, which consists of manifold vibro-roto energy sublevels (see Figure 1(a)). When a photon of suitable wavelength interacts with it, and gets absorbed, the molecule goes from the $S_{0}, v=0$, level to the electronically excited state $S_{1}, v=1,2,3$, level depending upon the Franck-Condon factor [2]. The molecule then undergoes thermalization by colliding with other molecules or the container walls and comes down to the $S_{1}, v=0$, level, which is the lowest vibrational state of the first excited state $\mathrm{S}_{1}$. It remains here for 5 to $10 \mathrm{~ns}$ and then goes down to the lower ground electronic state $S_{0}$ and any one of the vibrational states $v=1,2,3$, and so on, once again determined by the Franck-Condon factor. During this process, it may emit a photon (fluorescence) or may not do so (internal conversion). As the molecule has lost a substantial fraction of its excitation energy in the $S_{1}$ due to thermalization, the fluorescence occurs with longer wavelengths (or lower energy) than the incident light. As the $S_{1}$ and $S_{0}$ energy levels are quasi continuum, absorption and fluorescence can occur across a range of wavelengths (about 50-100 nm); also from the time of absorption, all the thermalizations occur in a picosecond time scale, while the fluorescence occurs in a nanosecond time scale, always starting from $\mathrm{S}_{1}, v=0$, independent of excitation wavelength; in other words, the fluorescence spectral profile is independent of the excitation wavelength, although the intensity is dependent. This is the Kasha-Vavilov rule [2].

Such absorption and reemission (fluorescence) are reasonably strong only in a few specific set of molecules, like dyes, which have a chain of alternate single and double levels, called conjugation. Most spectrofluorometers, commonly employed in research labs, are used only for the studies mentioned above.

In contrast, scattering is generally nonspecific, implying that light of any wavelength can interact with any molecule 


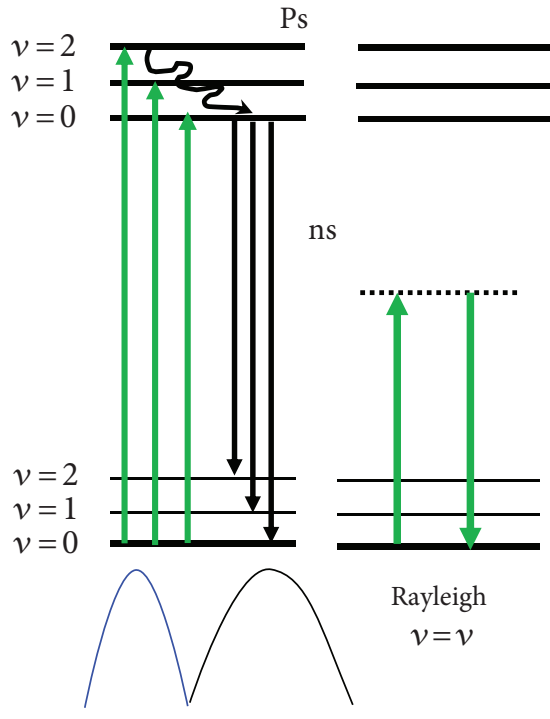

Absorption Fluorescence (b)

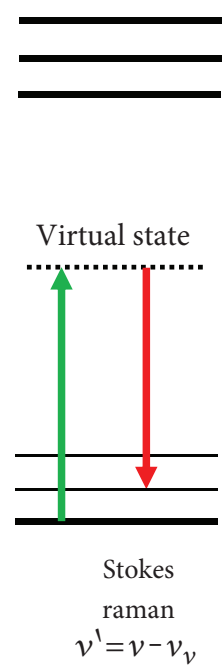

(c)

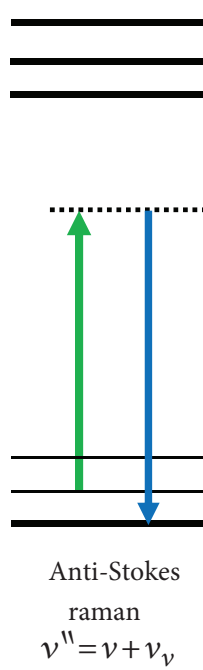

(d)

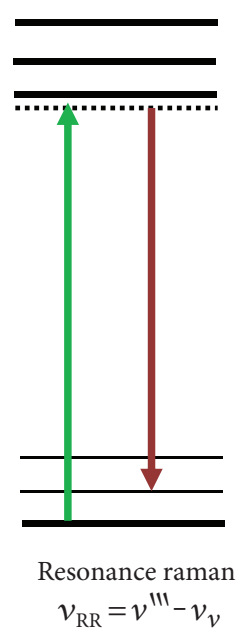

(e)

FIGURE 1: Schematic of energy level diagram of a molecule. $S_{0}$ and $S_{1}$ are electronic energy levels of a molecule, with vibration levels $v=0,1,2$. Rotational levels are within each vibrational level, but not shown here. (a) For Rayleigh scattering, (b) for Raman scattering Stokes, (c) for Raman scattering anti-Stokes, and (d) for resonance Raman scattering.

and undergo scattering. In this instance, if a photon of frequency $v$ interacts with a molecule in the $\mathrm{S}_{0}, v=0$, state, the molecule goes to a virtual state, a nonexistent state represented by a dotted line (Figure 1(b)) and comes back very quickly (in a picosecond time scale) to the $\mathrm{S}_{0}, v=0$, state. In this case, the photon does not lose any energy. The scattered photon has the same frequency $v$ as the incident one. This is called Rayleigh scattering. However, if the molecule returns to the $S_{1}, v=1$, state, (a vibrationally excited level) the scattered photon has less energy $\left(v^{\prime}=v-v_{v}\right.$, where $v_{v}$ is the frequency of the vibration of the molecule). This happens only occasionally; therefore, the scattered radiation of frequency $v^{\prime}=v-v_{v}$ is rather difficult to detect (see Figure 1(c)). There is one more possibility, the molecule may already be in a vibrationally excited state $\left(\mathrm{S}_{0}, v=1\right)$, and the incident photon of $v$ can move it to another virtual level (Figure 1(d)); when this comes down to the $\mathrm{S}_{0}, v=0$, level, the scattered photon has a higher energy and frequency of $v^{\prime \prime}=v+v_{v}$. The extra energy has been conferred by the molecule. As this is a very rare occurrence, the radiation with $v^{\prime \prime}=v+v_{v}$ is even more difficult to detect. Roughly, as a rule of thumb, if 100 photons produce Rayleigh scattering, only 10 will produce $v^{\prime}=v-v_{v}$ (called Stokes Raman) and only two will produce $v^{\prime}=v+v_{v}$ (called anti-Stokes Raman). An observed Indian scientist, Sir CV Raman, the first to detect such molecular scattering of radiation with quantized energy $v, v \pm v_{v}$, was awarded the Nobel Prize in 1930.

It is very significant that for a given molecule, both types of scattering can be observed by employing UV, VIS, or IR radiation, because the virtual level can be found anywhere. On the other hand, the shift in the scattered radiation $v-v^{\prime \prime}= \pm v_{v}$ is the characteristic fingerprint of the molecule, as its unique vibrational frequency is dependent on the configuration of the molecule. The whole of Raman spectroscopy, as an analytical tool, rests on this concept.

Scattering is always from a real quantum state to a virtual state whereas fluorescence is a phenomenon that occurs between two quantum states; hence, the latter is 10-100 times stronger than the former.

In order to obtain the Raman signal reasonably comparable to the fluorescence signal, one must resort to resonance Raman scattering $[3,4]$. In this instance, one must launch a radiation of wavelength $v$ that would raise the molecule from the real quantum state $S_{0}, v=0$, to a virtual state that almost overlaps the real quantum state of $S_{1}, v=0$, as shown in (Figure 1(e)). This could be done by groping and exploring the region between the absorption and fluorescence bands as evident from the following experiment.

Many new reviews shows ever increasing applications of Raman scattering in fields such as material science from bulk to nano. In nanotechnology, new techniques such as nano-Raman techniques based on aperture scanning near-field optical microscopy (SNOM) are maturing and diversifying [5], which enables researchers to do Raman studies at nano scales. In chemistry, it gives a thumbprint to identify the molecules [6-8]. The Raman spectroscopy has immense application in other fields such as clinical spectroscopy [9], biological samples analysis [10], and clinical instrumentation [11]. Resonance Raman scattering (RRS) is an important technique and is found in many applications [12].

\section{Experiment}

The PerkinElmer model LS 55 was employed throughout this experiment; however, any other commonly available 


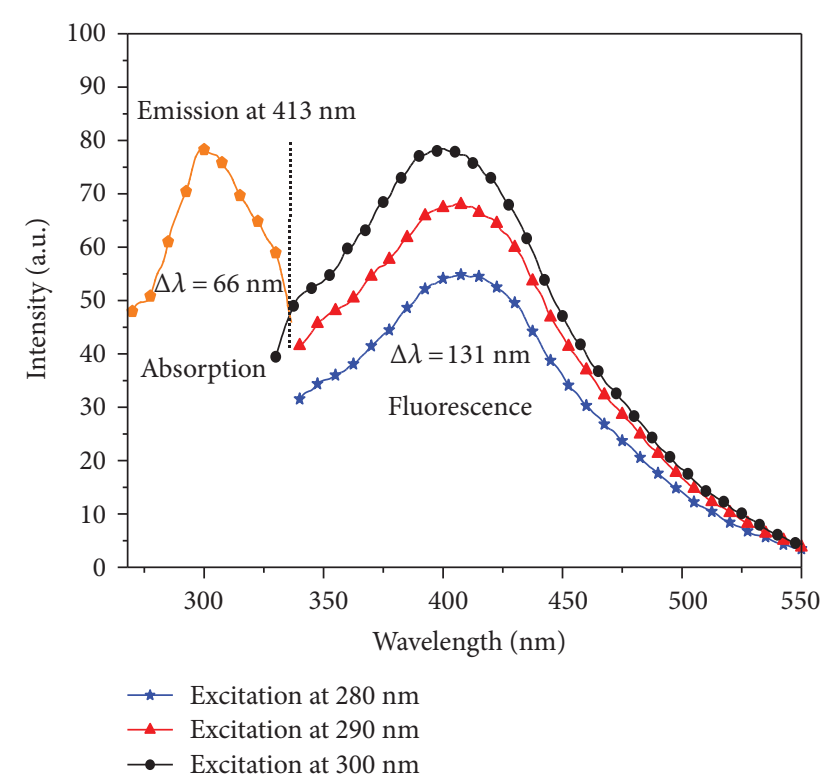

FIGURE 2: The fluorescence spectra of acetone with a peak at $413 \mathrm{~nm}$ for different wavelengths of excitation; on its left side is the excitation spectra with a peak at $327 \mathrm{~nm}$.

spectrofluorometer (Horiba, Hitachi, or Shimatsu), with dual gratings, one for excitation wavelength selection and another for emission band scanning, will be equally suitable.

2.1. Fluorescence Spectra. Acetone (analytical grade) quality was taken in a transparent quartz cuvette of $1 \mathrm{~cm}$ path length and loaded into the cell compartment of the instrument. Excitation wavelength was arbitrarily selected as $330 \mathrm{~nm}$; width was $10 \mathrm{~nm}$ in excitation as well as emission slits. The emission (synonymous with fluorescence in this case) spectrum was scanned from $350 \mathrm{~nm}$ to $600 \mathrm{~nm}$ and is presented in Figure 2, which shows a smooth band of emission with a peak at $413 \mathrm{~nm}$ of intensity 120 in the arbitrary units. This has a full width at half maximum (FWHM) of $102.4 \mathrm{~nm}$. Two more emission spectra were taken with excitation at 280 and $290 \mathrm{~nm}$, respectively. In both cases, the emission bands were similar, the only difference being the emission intensity was low. This confirms Kasha's rule (discussed earlier).

2.2. Excitation Spectra. In this case, the emission grating was fixed at $413 \mathrm{~nm}$, which was the emission peak of acetone and the excitation grating was rotated from $250 \mathrm{~nm}$ to $390 \mathrm{~nm}$ to obtain the excitation band of acetone, which had a peak at $327 \mathrm{~nm}$ with FWHM of $70 \mathrm{~nm}$. This corresponded to the $\mathrm{S}_{0}-\mathrm{S}_{1}$ absorption spectrum of acetone, obtainable from any UV-VIS spectrophotometer.

The excitation band indicates that if the excitation had been induced at $327 \mathrm{~nm}$, the emission peak would occur at $413 \mathrm{~nm}$, with the spectral profile of emission band becoming a mirror image of the excitation band, with the dotted line acting as the mirror as shown in Figure 2. For the other wavelengths of excitation, the emission intensity would be markedly low.

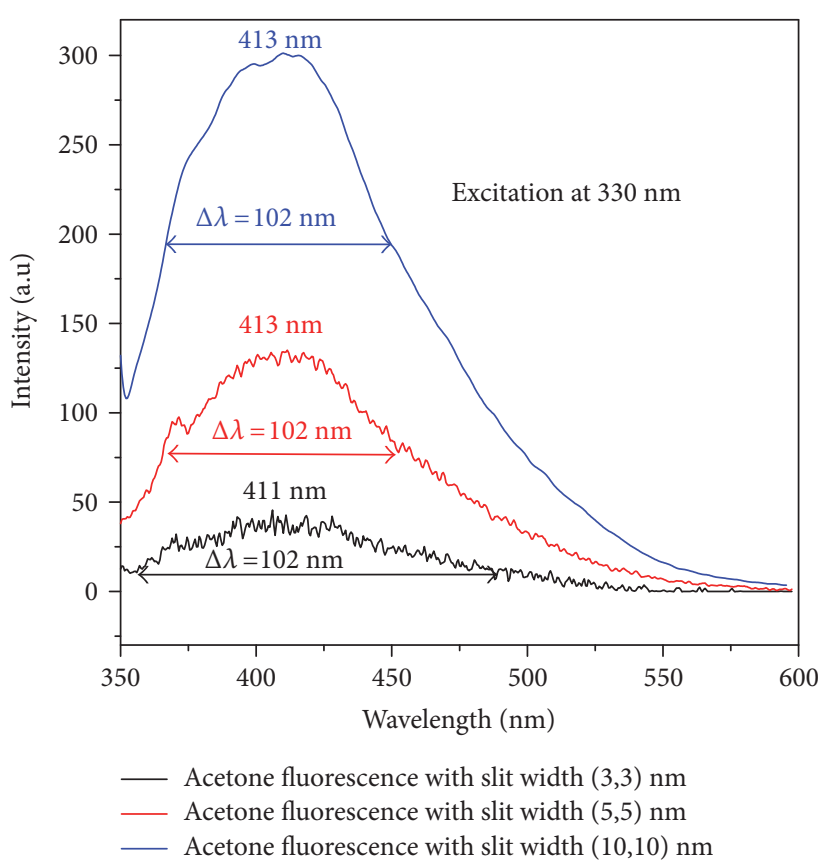

FIgURE 3: Acetone fluorescence with different slit widths.

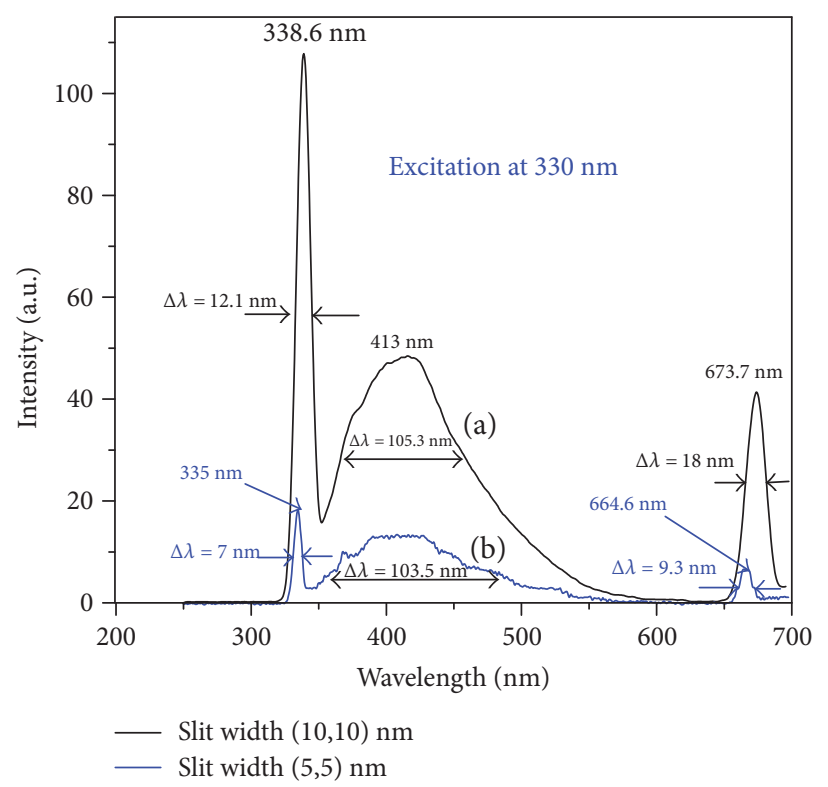

FIGURE 4: Rayleigh scattering and fluorescence signal with different slit widths (for acetone). (a) Black line and (b) blue line.

2.3. Fluorescence Spectra for Different Slit Widths. Utilizing the same acetone sample and excitation at $330 \mathrm{~nm}$, emission spectra were obtained for 10,5 , and $3 \mathrm{~nm}$ spectral widths, in excitation as well as emission slits. The spectra in Figure 3 shows the same FWHM of $102 \mathrm{~nm}$ in each case; only the intensity has decreased twice or thrice. All the features mentioned above would be markedly different for scattering spectra.

2.4. Rayleigh Scattering. To obtain the Rayleigh scattering spectra of acetone, the excitation grating was fixed at $330 \mathrm{~nm}$; 


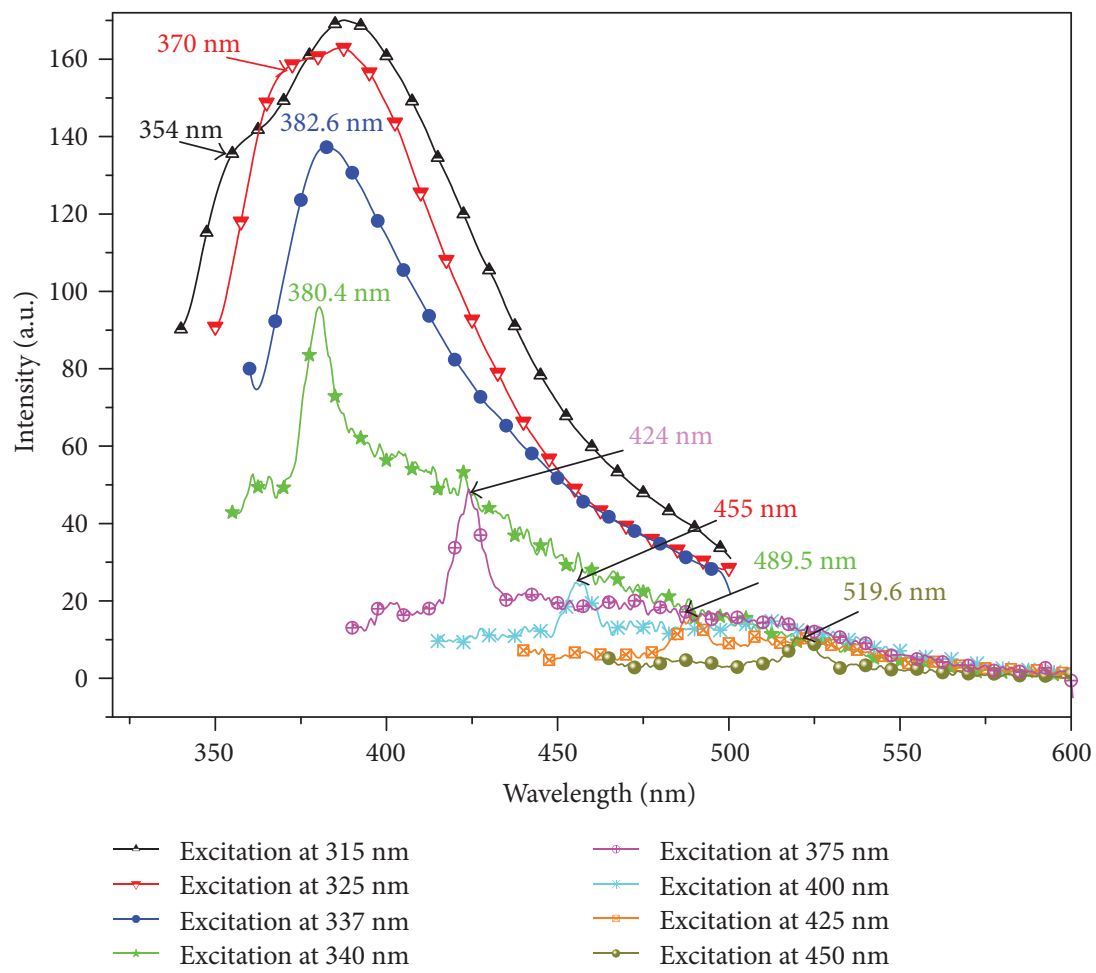

FIgURE 5: Resonance Raman spectra for acetone.

TABle 1: Resonance Raman spectra of acetone.

\begin{tabular}{|c|c|c|c|c|c|c|}
\hline Number & $\begin{array}{l}\text { Excitation wavelength } \\
\qquad \lambda_{e}(\mathrm{~nm})\end{array}$ & $\begin{array}{l}\text { Excitation wave number } \\
\qquad v_{e}\left(\mathrm{~cm}^{-1}\right)\end{array}$ & $\begin{array}{c}\text { Raman signal } \\
\text { In wavelength } \\
\lambda_{R}(\mathrm{~nm}) \\
\end{array}$ & $\begin{array}{c}\text { Raman signal } \\
\text { In wave number } \\
v_{R}\left(\mathrm{~cm}^{-1}\right)\end{array}$ & $\begin{array}{c}\text { Raman shift } \\
v_{v}\left(\mathrm{~cm}^{-1}\right)\end{array}$ & $\begin{array}{c}\text { Intensity } \\
\text { (a.u.) }\end{array}$ \\
\hline 1 & 450 & 22222 & 519.6 & 19246 & 2976 & 10.7 \\
\hline 2 & 425 & 23529 & 489.5 & 20429 & 3100 & 17 \\
\hline 3 & 400 & 25000 & 455 & 21978 & 3022 & 25.5 \\
\hline 4 & 375 & 26667 & 424 & 23585 & 3082 & 49 \\
\hline 5 & 340 & 29412 & 380.4 & 26288 & 3124 & 95 \\
\hline 6 & 337 & 29673 & 382.6 & 26136 & 3537 & 138 \\
\hline 7 & 325 & 30769 & 370 & 27027 & 3742 & 158 \\
\hline 8 & 315 & 31746 & 354 & 28248 & 3498 & 135.3 \\
\hline Average & & & & & $3260 \pm 10$ & \\
\hline
\end{tabular}

the slit width was $10 \mathrm{~nm}$ in each slit and the emission grating was scanned from $200 \mathrm{~nm}$ to $700 \mathrm{~nm}$. The spectrum, as shown in Figure 4(a), indicates a sharp peak at $338 \mathrm{~nm}$ with $\mathrm{FWHM}=12 \mathrm{~nm}$, another broad band at $410 \mathrm{~nm}$, and the next sharp peak at $673 \mathrm{~nm}(\mathrm{FWHM}=18 \mathrm{~nm})$.

The first band, without experiencing any change in wavelength, is the Rayleigh scattering (please note, it should have occurred at $330 \mathrm{~nm}$ but instead was seen at $338 \mathrm{~nm}$ due to instrumental artifacts); the $410 \mathrm{~nm}$ band is the fluorescence band of acetone; the band at $673 \mathrm{~nm}$ is due to the Rayleigh scattering occurring in the second order of grating (this is an experimental unavailable artifact; if only a prism had been used instead of grating for dispersion, it would have disappeared).

For the same sample and scattering setup arrangement, the experiment was repeated with slit widths $=5 \mathrm{~nm}$ in each.
As shown in Figure 4(b), the spectra of Figure 4(a and b) are very similar barring two major differences. The intensity in all the bands had decreased (as expected); but the FWHM of Rayleigh scattering also dropped from $12 \mathrm{~nm}$ to $7 \mathrm{~nm}$. The fluorescence spectra of acetone, however, continued to remain at $102 \mathrm{~nm}$. This is the first major difference between scattering and fluorescence.

Scattering is, in fact, an irregular reflection at the molecular surface, very similar to a tennis ball bouncing off a racquet. What comes, goes off in a different direction, with minimal or no interaction. In contrast, during fluorescence, an incident photon is absorbed, and the energy gets thermalized in a few picoseconds and is then given off by the molecule, with its fingerprint, within the time span of a few nanoseconds. 


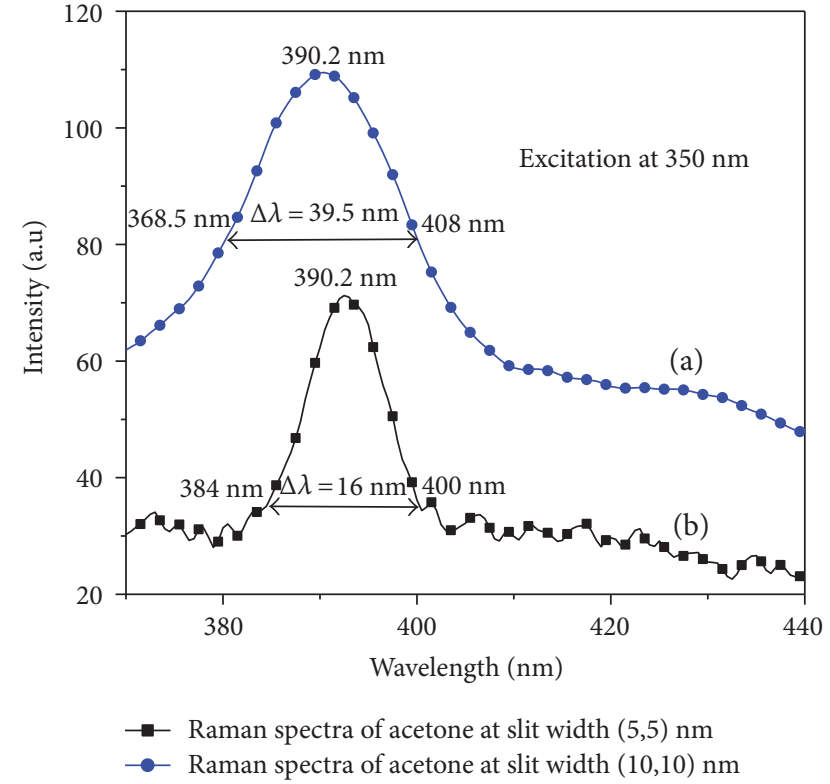

FIGURE 6: Raman spectra of acetone at different slit widths. (a) Blue line and (b) black line.

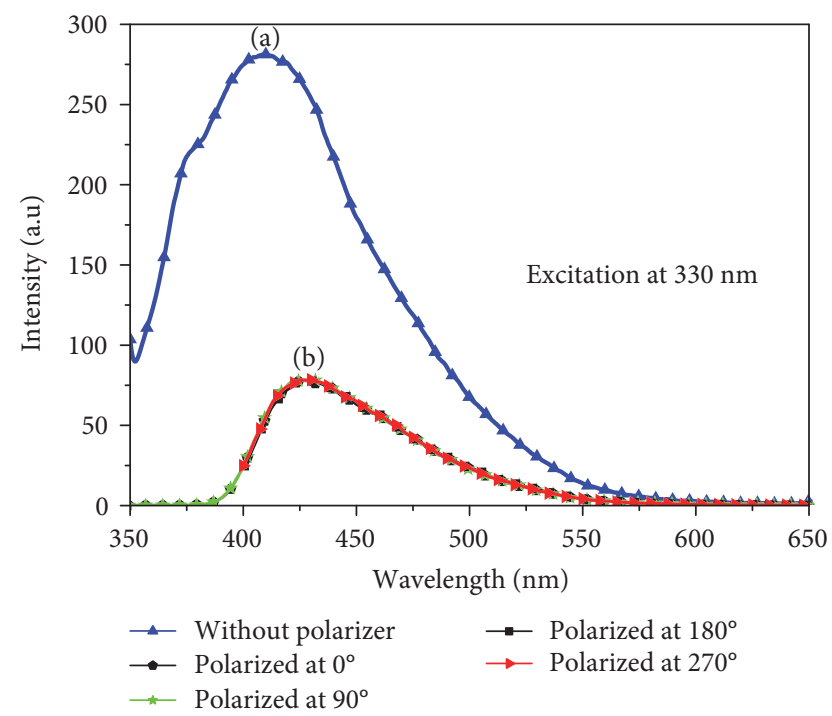

Figure 7: Acetone fluorescence spectra for different positions of polarizer.

2.5. Resonance Raman Scattering. For measuring the Raman spectral features of acetone, the excitation wavelength was fixed at $450 \mathrm{~nm}$ and the emission grating was scanned from $485 \mathrm{~nm}$ to $600 \mathrm{~nm}$. As evident from Figure 5, a small sharp signal was observed at $519.6 \mathrm{~nm}$, corresponding to the Raman shift of $(1 / 450 \mathrm{~nm}-1 / 519.6 \mathrm{~nm})=2976 \mathrm{~cm}^{-1}$. This $2976 \mathrm{~cm}^{-1}$ represents the $\mathrm{C}-\mathrm{H}$ bond vibrational frequency of the acetone molecule which causes the Raman shift.

To confirm this, the experiment was repeated with the excitation at $425 \mathrm{~nm}$ and scanning was done from $440 \mathrm{~nm}$ to $600 \mathrm{~nm}$. The Raman signal now appeared at $489.5 \mathrm{~nm}$ and the Raman shift was $(1 / 425 \mathrm{~nm}-1 / 489.5 \mathrm{~nm})=3100 \mathrm{~cm}^{-1}$.

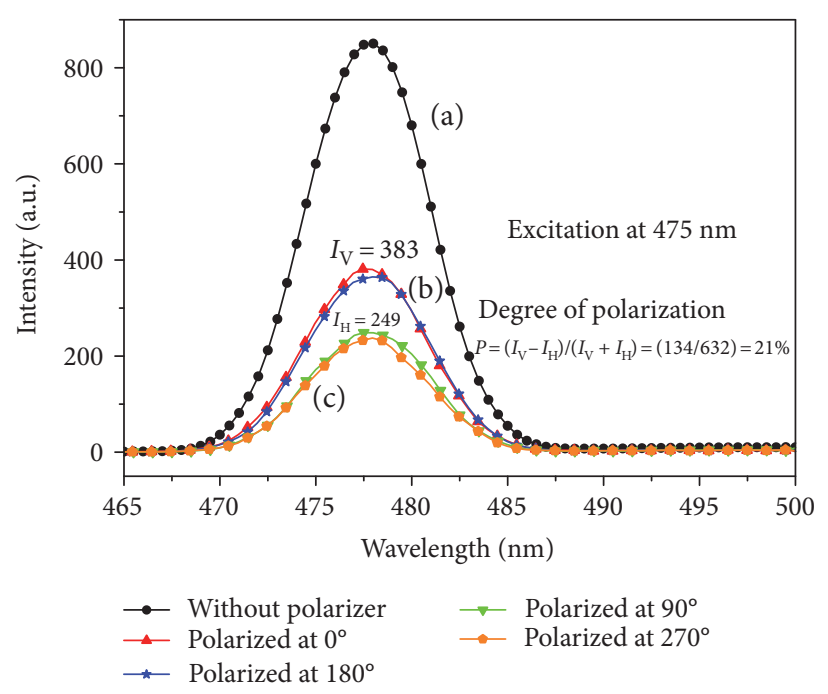

Figure 8: Polarization of Rayleigh scattering of acetone. (a) Black line; (b) blue and red line; (c) green and orange line.

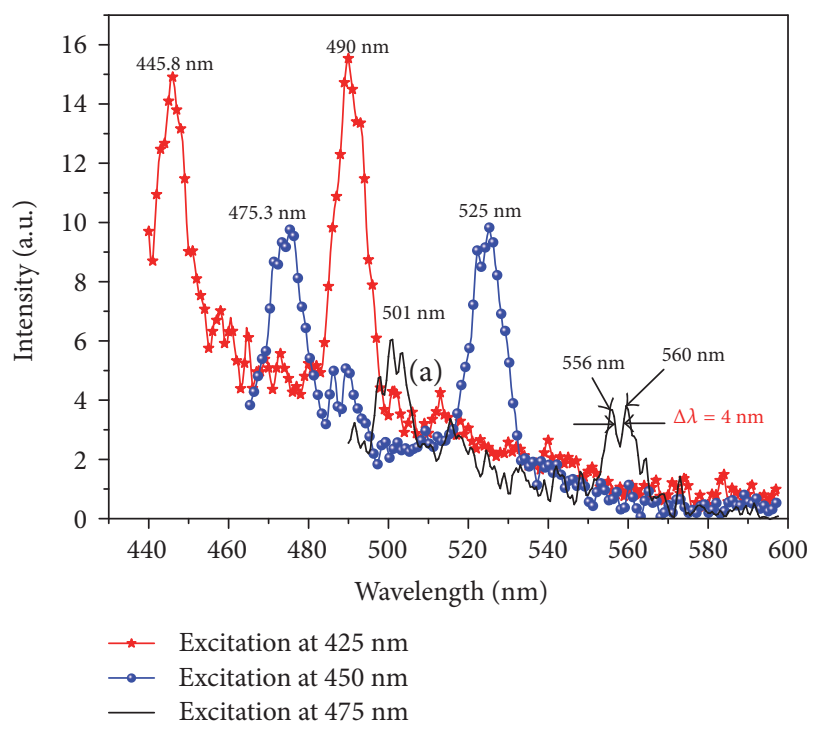

FIgURE 9: Raman spectra of benzene at different excitation wavelengths. (a) is black line.

This implies that the Raman shift is a constant and characteristic of the molecule, but the Raman signal moves with the incident light. The experiment was repeated by shifting the excitation to 400,375 , and $340 \mathrm{~nm}$. In each case, the Raman scattered signal got shifted to 455,424 , and $380 \mathrm{~nm}$; but the shift in their wave number approximately corresponded to $3060 \mathrm{~cm}^{-1}$, confirming the Raman effect.

The most significant feature of this part of the experiment is as follows: as the excitation was moved from $450 \mathrm{~nm}$ to $315 \mathrm{~nm}$, over a range of $135 \mathrm{~nm}$, the Raman signal intensity changed from 9 to 160 (in arbitrary unit), an increase by 17 times. Apparently, $340 \mathrm{~nm}$ represents the near resonance Raman spectra of acetone. When the excitation goes to $325 \mathrm{~nm}$, the Raman signal occurring at $350 \mathrm{~nm}$ was 
TABLE 2: Raman spectra for benzene.

\begin{tabular}{|c|c|c|c|c|c|c|}
\hline Number & $\begin{array}{c}\text { Excitation wavelength } \\
\lambda_{e}(\mathrm{~nm})\end{array}$ & $\begin{array}{l}\text { Excitation wave number } \\
\qquad v_{e}\left(\mathrm{~cm}^{-1}\right)\end{array}$ & $\begin{array}{c}\text { Raman signal } \\
\text { In wavelength } \\
\lambda_{R}(\mathrm{~nm}) \\
\end{array}$ & $\begin{array}{c}\text { Raman signal } \\
\text { In wave number } \\
v_{R}\left(\mathrm{~cm}^{-1}\right)\end{array}$ & $\begin{array}{c}\text { Raman shift } \\
v_{v}\left(\mathrm{~cm}^{-1}\right)\end{array}$ & $\begin{array}{l}\text { Intensity } \\
\text { (a.u.) }\end{array}$ \\
\hline \multirow{2}{*}{1} & \multirow{2}{*}{475} & \multirow{2}{*}{21053} & 501 & 19960 & 1093 & 6 \\
\hline & & & 556 & 17986 & 3067 & 3.7 \\
\hline \multirow{2}{*}{2} & \multirow{2}{*}{450} & \multirow{2}{*}{22222} & 475.3 & 21039 & 1183 & 9.9 \\
\hline & & & 525 & 19048 & 3174 & 9.8 \\
\hline \multirow{2}{*}{3} & \multirow{2}{*}{425} & \multirow{2}{*}{23529} & 445.8 & 22472 & 1057 & 14.9 \\
\hline & & & 490 & 20408 & 3121 & 15.5 \\
\hline
\end{tabular}

comparable in intensity to the fluorescence at $413 \mathrm{~nm}$, representing the resonance Raman excitation; for excitation at 315 , the Raman signal occurred at $352 \mathrm{~nm}$, with the intensity of 120. For excitations with a wavelength lower than this, the acetone produced only a fluorescent broad band with a peak of $413 \mathrm{~nm}$, as discussed earlier. Or, in other words, the best region to obtain resonance Raman is the valley between the excitation and emission bands.

Table 1 summarizes the results mentioned above. (Note also, the Raman shift gradually changed from 2970 to $3500 \mathrm{~cm}^{-1}$ as the excitations moved from longer to shorter wavelengths due to certain molecular properties.)

2.6. Raman Spectra for Different Slit Widths. The next experiment was performed with reduced slit widths. Figure 6 shows the Raman spectra with excitation at $350 \mathrm{~nm}$, which produced a signal at $390 \mathrm{~nm}$. In Figure 6(a), slit width $10 \mathrm{~nm}$ (in excitation and emission slits) is shown, and Figure 6(b) shows the spectra with slit width $5 \mathrm{~nm}$ each. As the slit widths were reduced, the intensity of signal was observed to decrease; more important was the drop in the FWHM from $39.5 \mathrm{~nm}$ to $16 \mathrm{~nm}$. This is again very characteristic of any scattering phenomena, regardless of whether Rayleigh or Raman.

In fact, when the Nd:YAG laser (at $532 \mathrm{~nm}$ ) was used to produce Raman signal from acetone, a sharp signal at $630 \mathrm{~nm}$ of FWHM of $1 \mathrm{~nm}$ or less was obtained [13, 14]; but when Rhodamine dye solution was excited, a broad fluorescence was observed with the peak at $630 \mathrm{~nm}$ and FWHM of $20 \mathrm{~nm}$ [15].

2.7. Polarization Properties. Another interesting experiment was performed to highlight the differences in the polarization properties.

Figure 7 shows the fluorescence of acetone when excited at $330 \mathrm{~nm}$. Figure 7 (a) reveals the fluorescence without any polarizer; whereas Figure 7(b) shows the fluorescence with the polarizer maintained at $0,90,180$, and 270 degrees of orientation. (The polarizer was inserted between the emission slit and detector.) It is very obvious that the polarizer by itself has cut off the signal by four times due to the absorption by the material around 350 to $600 \mathrm{~nm}$ but such a reduction was independent of the orientation of the polarizer proving that the fluorescence from the molecules in the solution get depolarized. The fluorescence is a process involving three steps, the absorption of a photon as a

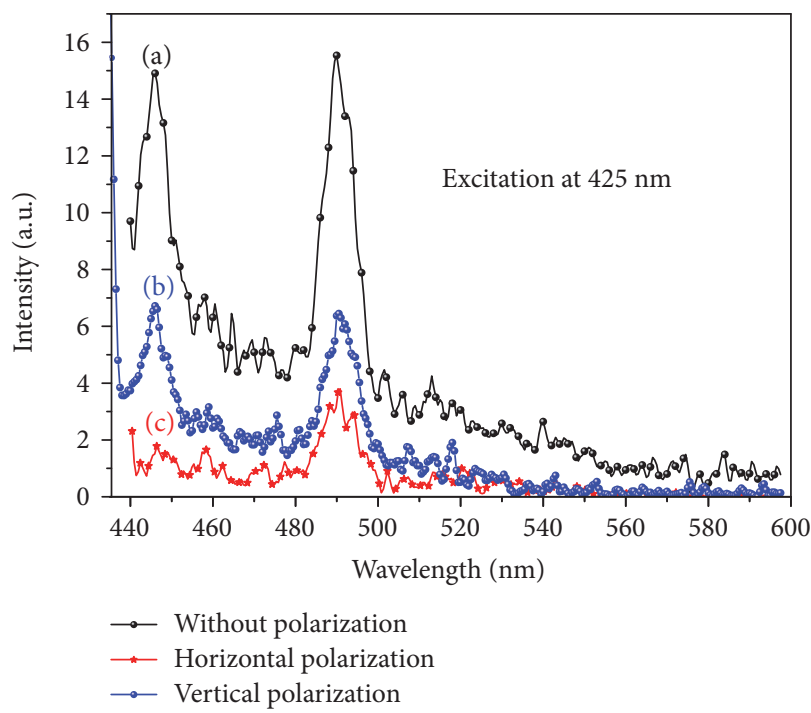

FIgURE 10: Polarized Raman spectra for benzene.

whole, thermalization, and reemission; the polarization feature is generally lost, particularly in a liquid medium where the molecules are in a state of constant collision among themselves.

Figure 8 shows the Rayleigh scattering signal for the excitation of acetone at $475 \mathrm{~nm}$. The resultant signal occurred at $478 \mathrm{~nm}$ (due to experimental artifacts). Figure 8(a) shows a signal of 800 units without any polarizer. In Figure $8(\mathrm{~b})$, the signal is obvious with the polarizer at 0 and 180 degrees and an intensity of 383 ; Figure 8 (c) shows the signal of intensity 249 for the polarizer at 90 and 270 degrees. Thus, it is clearly evident that the degree of polarization for the Rayleigh scattering is 0.21 .

Figure 9(a) shows the Raman spectra drawn for benzene, with excitation at $475 \mathrm{~nm}$. The Raman signals occurred at $501 \mathrm{~nm}$ and $560 \mathrm{~nm}$, which corresponded to $v_{v}=1100 \mathrm{~cm}^{-1}$ and $3010 \mathrm{~cm}^{-1}$, due to skeleton breathing and the stretching of the benzene $\mathrm{C}-\mathrm{H}$ bond. This is again confirmed by exciting at $450 \mathrm{~nm}$ and observing the two Raman signals mentioned above, at 475.3 and $525 \mathrm{~nm}$. Another excitation was with the $425 \mathrm{~nm}$ produced Raman signals at 445.8 and $490 \mathrm{~nm}$. (The instrument is so sensitive that even the rotational features of the Raman spectra were observable; 
e.g., the Raman signal for excitation at $475 \mathrm{~nm}$ produced two lines at 556 and $560 \mathrm{~nm}$, a separation of $18 \mathrm{~cm}^{-1}$; however, it got blurred for the other excitation wavelengths.) Table 2 summarizes the results given above.

Figure 10(a) shows the Raman signal of benzene as excited at $425 \mathrm{~nm}$, without any polarizer. Figure 10(b) shows the same with the polarizer placed horizontally, and Figure 10(c) shows the same with the polarizer rotated to $90^{\circ}$. Both bands are clearly polarized differently. The $1100 \mathrm{~cm}^{-1}$ band appears more strongly polarized than the other, with the degrees of polarization $p=0.6$ and 0.2 , respectively.

It is noteworthy that all scattering exhibits a high degree of polarization, because it changes both direction and polarization.

\section{Conclusion}

Utilizing a spectrofluorometer commonly available in any graduate laboratory, a distinction between most of the spectral features of fluorescence and scattering has been clearly demonstrated (Rayleigh and Raman). It has elegantly been shown that the valley between the two mountains of absorption and emission is the best region to grope for the resonance Raman spectra. It has also been shown that the scattered signals are strongly polarized and that their spectral widths are determined most often by the width of the incident signal. In contrast, fluorescence is neither polarized nor dependent on the incident signal. Further, scattering is many times more common than fluorescence in general; however, for a few select, conjugated molecules like dyes, the fluorescence is tremendously high that it is almost impossible to observe the scattering signals. On the other hand, in the weakly fluorescent molecules like acetone or benzene, the scattering signals are comparable to, and sometimes even higher than, the fluorescence.

\section{Conflicts of Interest}

The authors declare that they have no conflicts of interest.

\section{Acknowledgments}

The authors would like to extend their sincere appreciation to the Deanship of Scientific Research at King Saud University for its funding of this research through the Research Group Project no. "RGP-223."

\section{References}

[1] C. V. Raman and K. S. Krishnan, "A new type of secondary radiation," Nature, vol. 121, pp. 501-502, 1928.

[2] M. Kasha, "Characterization of electronic transitions in complex molecules," Discussions of the Faraday Society, vol. 9, pp. 14-19, 1950.

[3] P. M. Kroneck and M. Sosa Torres, Sustaining Life on Planet Earth: Metalloenzymes Mastering Dioxygen and Other Chewy Gases, Springer International Publishing, Switzerland, 2015.

[4] S. Hu, K. M. Smith, and T. G. Spiro, "Assignment of protoheme resonance Raman spectrum by heme labeling in myoglobin," Journal of the American Chemical Society, vol. 118, no. 50, pp. 12638-12646, 1996.

[5] R. C. Prince, R. R. Frontiera, and E. O. Potma, "Stimulated Raman scattering: from bulk to nano," Chemical Reviews, 2016.

[6] N. Jiang, N. Chiang, L. R. Madison et al., "Nanoscale chemical imaging of a dynamic molecular phase boundary with ultrahigh vacuum tip-enhanced Raman spectroscopy," Nano Letters, vol. 16, no. 6, pp. 3898-3904, 2016.

[7] S. Nie and S. R. Emory, "Probing single molecules and single nanoparticles by surface-enhanced Raman scattering," Science, vol. 275, no. 5303, pp. 1102-1106, 1997.

[8] C. L. Evans, E. O. Potma, M. Puoris' Haag, D. Côté, C. P. Lin, and X. S. Xie, "Chemical imaging of tissue in vivo with videorate coherent anti-stokes Raman scattering microscopy," Proceedings of the National Academy of Sciences of the United States of America, vol. 102, no. 46, pp. 16807-16812, 2005.

[9] O. Stevens, I. E. I. Petterson, J. C. Day, and N. Stone, “Developing fibre optic Raman probes for applications in clinical spectroscopy," Chemical Society Reviews, vol. 45, no. 7, pp. 1919-1934, 2016.

[10] S. Laing, K. Gracie, and K. Faulds, "Multiplex in vitro detection using SERS," Chemical Society Reviews, vol. 45, no. 7, pp. 1901-1918, 2016.

[11] I. Pence and A. Mahadevan-Jansen, "Clinical instrumentation and applications of Raman spectroscopy," Chemical Society Reviews, vol. 45, no. 7, pp. 1958-1979, 2016.

[12] B. Robert, "Resonance Raman spectroscopy," Photosynthesis Research, vol. 101, no. 2-3, pp. 147-155, 2009.

[13] A. M. Azzeer and V. Masilamani, "A new, efficient laser source at $630 \mathrm{~nm}$ for photodynamic therapy and pulsed hologram," Japanese Journal of Applied Physics, vol. 42, no. 2R, p. 471, 2003.

[14] V. Masilamani and A. Aldwayyan, "Evidence of superexciplex in dye molecules," Japanese Journal of Applied Physics, vol. 41, no. 9R, p. 5801, 2002.

[15] R. Pratesi, Optronic Techniques in Diagnostic and Therapeutic Medicine, Springer Science \& Business Media, 2012. 

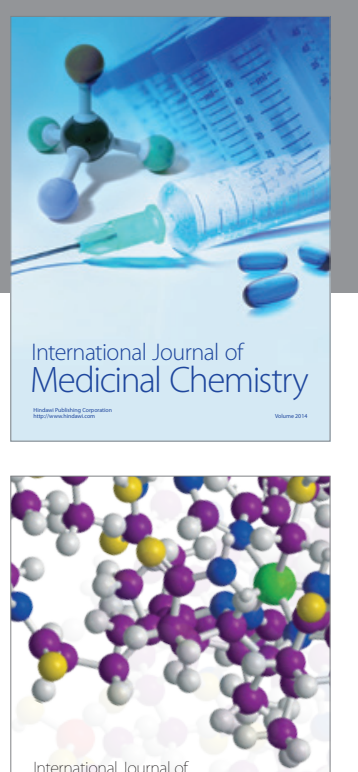

Carbohydrate Chemistry

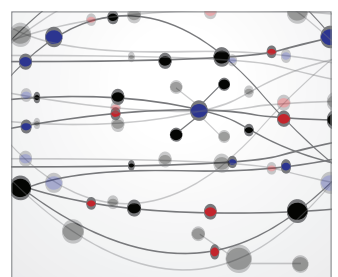

The Scientific World Journal
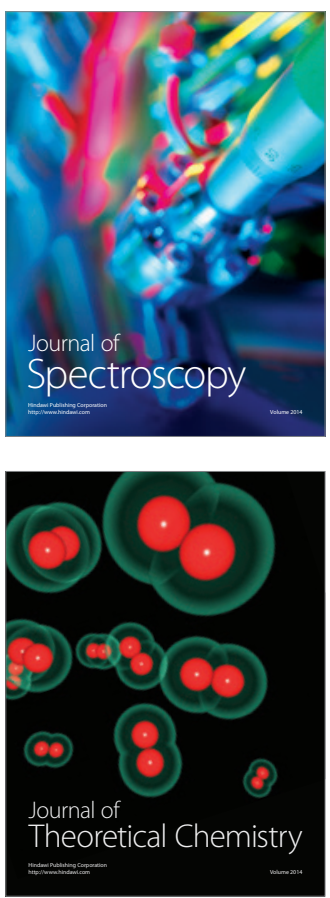
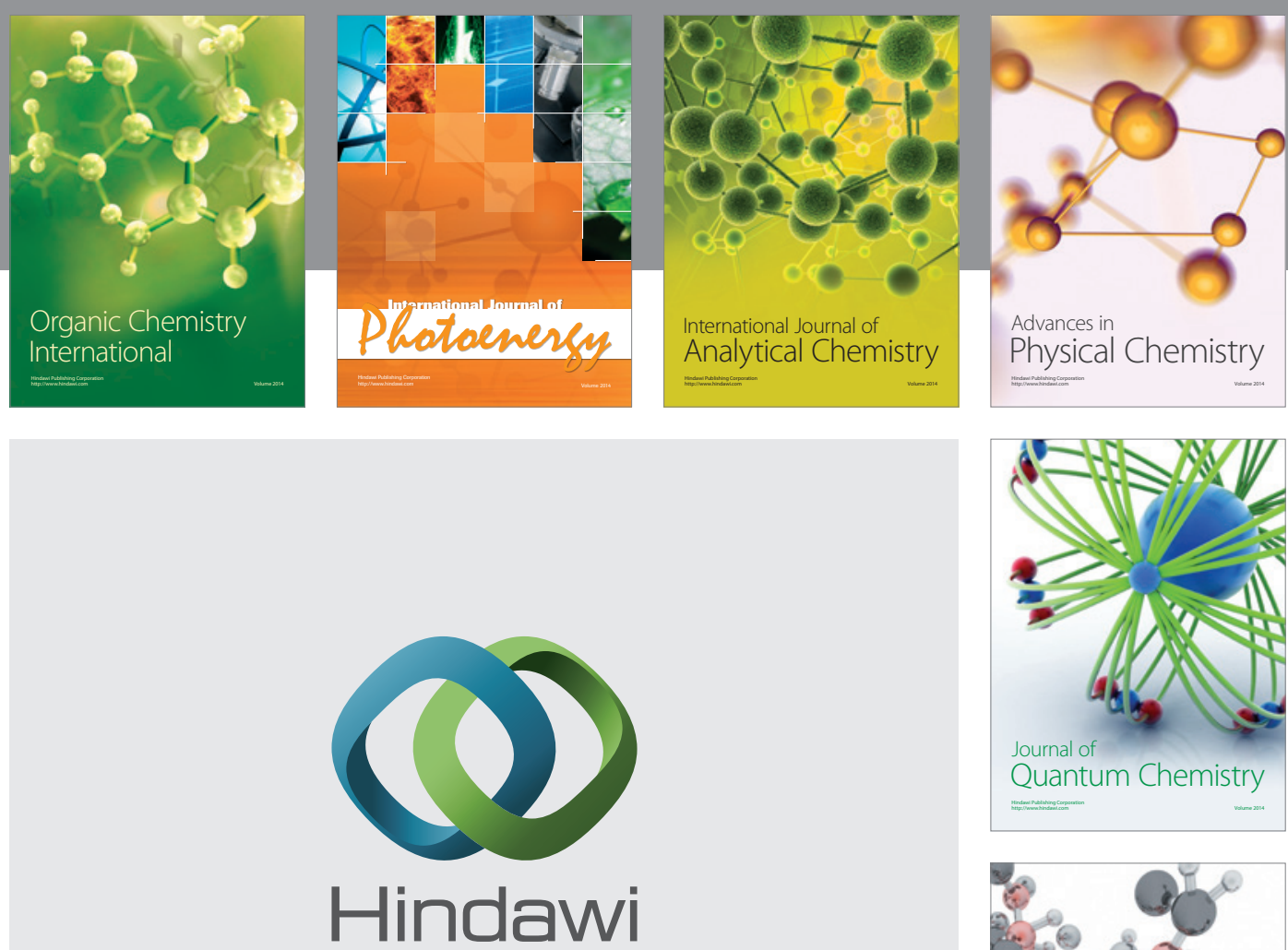

Submit your manuscripts at

https://www.hindawi.com

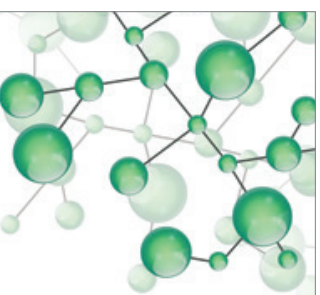

International Journal of

Inorganic Chemistry
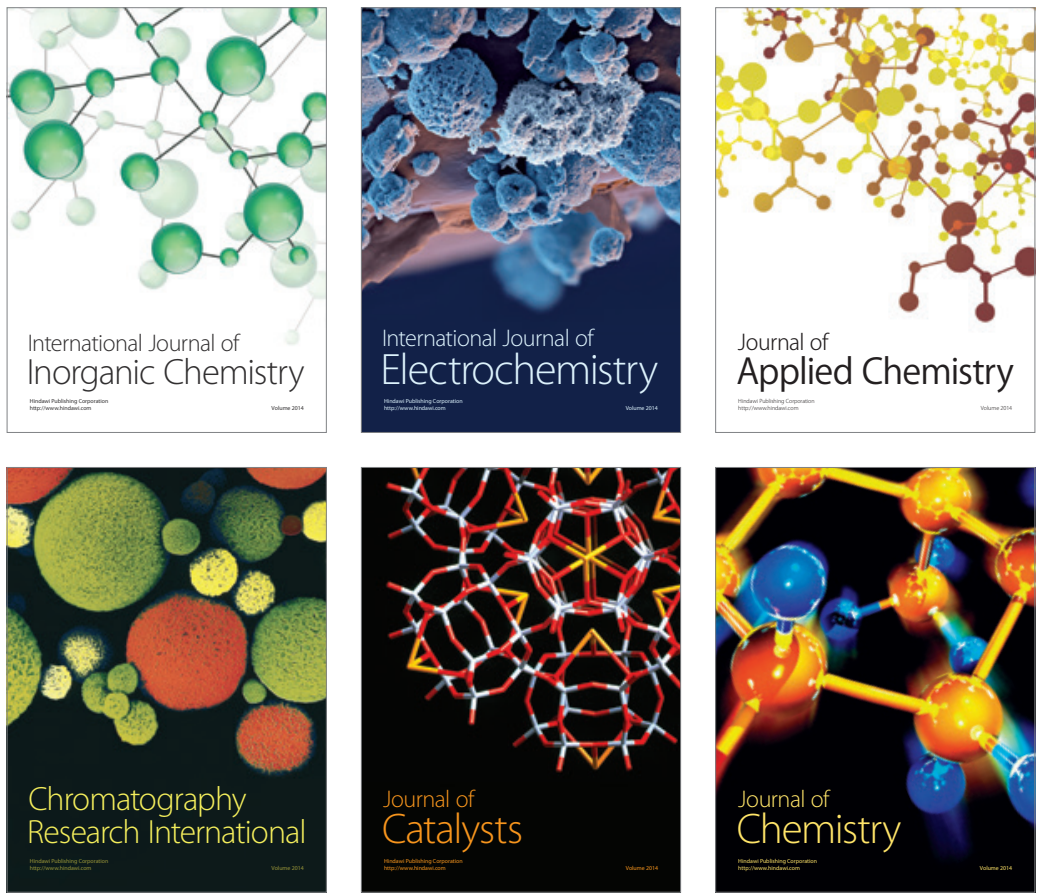

Journal of

Applied Chemistry
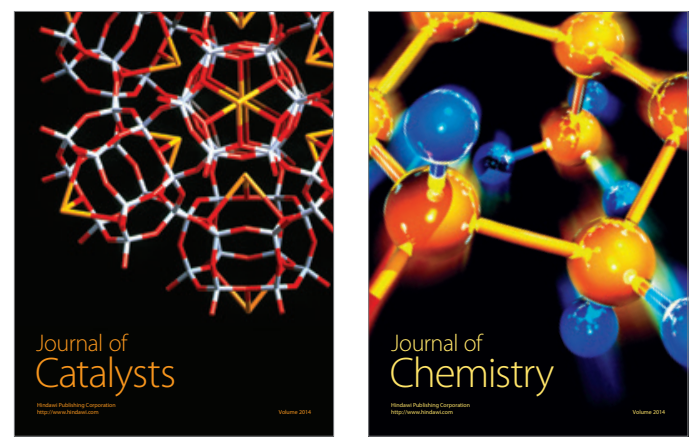
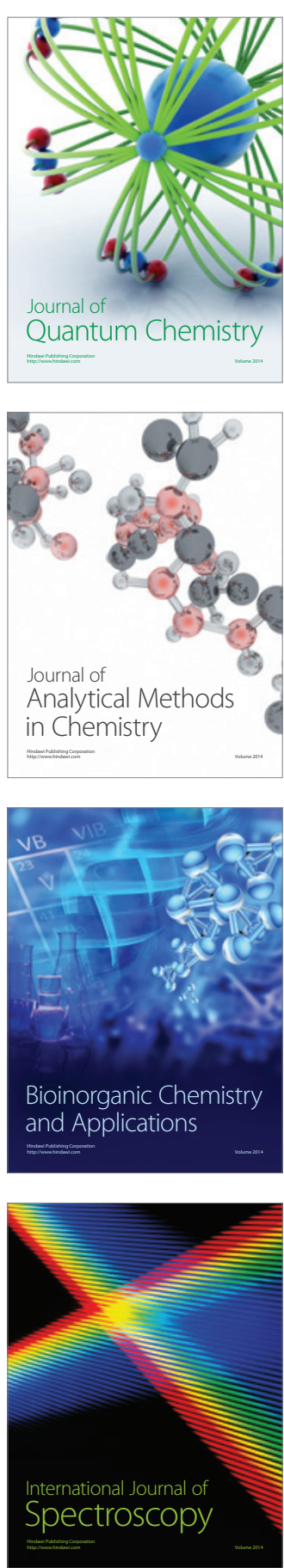